\title{
A biharmonic equation with singular nonlinearity
}

\author{
Marius Ghergu \\ School of Mathematical Sciences, \\ University College Dublin, \\ Belfield, Dublin 4, Ireland \\ E-mail: marius.ghergu@ucd.ie
}

September 21, 2018

\begin{abstract}
We study the biharmonic equation $\Delta^{2} u=u^{-\alpha}, 0<\alpha<1$, in a smooth and bounded domain $\Omega \subset \mathbb{R}^{n}, n \geq 2$, subject to Dirichlet boundary conditions. Under some suitable assumptions on $\Omega$ related to the positivity of the Green function for the biharmonic operator, we prove the existence and uniqueness of a solution.
\end{abstract}

Keywords Biharmonic operator; singular nonlinearity; Green function; integral equation

Mathematics Subject Classifications (2000) 35J40; 35J08; 45G05; 47H10

\section{Introduction and the main results}

In this paper we study the biharmonic elliptic problem

$$
\begin{cases}\Delta^{2} u=u^{-\alpha}, u>0 & \text { in } \Omega, \\ u=\partial_{\nu} u=0 & \text { on } \partial \Omega,\end{cases}
$$

where $0<\alpha<1, \Omega \subset \mathbb{R}^{n}(n \geq 2)$ is a smooth bounded domain in the sense that we will describe in the following, $\nu$ is the exterior unit normal at $\partial \Omega$ and $\partial_{\nu}=\frac{\partial}{\partial \nu}$ is the outer normal derivative at $\partial \Omega$. We denote by $G(\cdot, \cdot)$ the Green function associated with the biharmonic operator $\Delta^{2}$ subject to Dirichlet boundary conditions, that is, for all $y \in \Omega, G(\cdot, y)$ satisfies in the distributional sense:

$$
\begin{cases}\Delta^{2} G(\cdot, y)=\delta_{y}(\cdot) & \text { in } \Omega, \\ G(\cdot, y)=\partial_{\nu} G(\cdot, y)=0 & \text { on } \partial \Omega .\end{cases}
$$

The study of Green function for the biharmonic equation goes back to Boggio [2] in 1901. He proved that the Green function is positive in any ball of $\mathbb{R}^{n}$. Boggio [3] and Hadamard [17] conjectured that this fact should be true at least in any smooth convex domain of $\mathbb{R}^{n}$.

Starting with the late 1940s, various counterexamples have been constructed that disprove the Boggio-Hadamard conjecture. For instance, if a domain in $\mathbb{R}^{2}$ has a right-angle, then the associated Green function fails to be everywhere positive (see Coffman and Duffin [4]). A similar result holds for thin ellipses: Garabedian [8] found that in an ellipse in $\mathbb{R}^{2}$ with the ratio of the half axes $\simeq 2$, the Green function for the biharmonic operator changes sign (for an elementary proof, see also Shapiro-Tegmark [21]). In turn, if the ellipse is close to a ball in the plane, Grunau and Sweers [12] proved that the Green function is positive. Recently, 
Grunau and Sweers $[13,14,15]$, Grunau and Robert [16] provided interesting characterizations of the regions where the Green function is negative. They also obtained that if a domain is sufficiently close to the unit ball in a suitable $C^{4, \gamma}$-sense, then the biharmonic Green function under Dirichlet boundary condition is positive.

It is worth noting here that the positivity property of the Green function for the biharmonic operator is a special feature of the prescribed boundary condition. Indeed, if instead of Dirichlet boundary condition one assumes Navier boundary condition (that is, $u=\Delta u=0$ on $\partial \Omega$ ), then a straightforward application of the second order comparison principle yields the positivity of the Green function. However, even under Navier conditions there is in general no positivity result for the Green function when the biharmonic operator is perturbed (see, e.g., [5, 18]).

In this paper we assume that $\Omega \subset \mathbb{R}^{n}, n \geq 2$, is a bounded domain that satisfies:

(A1) the boundary $\partial \Omega$ is of class $C^{16}$ if $n=2$ and of class $C^{12}$ if $n \geq 3$;

$(A 2)$ the Green function $G(\cdot, \cdot)$ is positive.

The assumption $(A 1)$ on the regularity of $\partial \Omega$ goes back to Krasovskii [19] and is taken from Dall'Acqua and Sweers [6] where sharp upper bounds for the Green function are obtained. The need for condition $(A 2)$ will become more clear once we specify what it is understood by a solution of (1.1). We say that $u$ is a solution of (1.1) if

$$
u \in C(\bar{\Omega}), \quad u>0 \quad \text { in } \Omega,
$$

and $u$ satisfies the integral equation

$$
u(x)=\int_{\Omega} G(x, y) u^{-\alpha}(y) d y \quad \text { for all } x \in \Omega .
$$

Also remark that condition $(A 2)$ above implies the standard maximum principle for the biharmonic operator in $\Omega$.

Although there are several results for biharmonic equations involving exponential or powertype nonlinearities with positive exponents $[1,7,9,10]$, to the best of our knowledge, there is no such a study for (1.1). Our main result concerning (1.1) is the following.

Theorem 1.1 Assume $0<\alpha<1$ and conditions (A1), (A2) hold. Then, the problem (1.1) has a unique solution $u$ and there exist $c_{1}, c_{2}>0$ such that

$$
c_{1} \delta^{2}(x) \leq u(x) \leq c_{2} \delta^{2}(x) \quad \text { in } \Omega,
$$

where $\delta(x)=\operatorname{dist}(x, \partial \Omega)$. Moreover, $u \in C^{2}(\bar{\Omega})$ and if $0<\alpha<1 / 2$ then $u \in C^{3}(\bar{\Omega})$.

The existence of a solution will be obtained by means of Schauder fixed point theorem. To this aim, we employ the sharp estimates for Green function given in [6]. The uniqueness relies heavily on the boundary estimate (1.3) which is obtained by using the behavior of the Green function (see Proposition 2.2 below).

The remaining part of the paper is organized as follows. In Section 2 we derive some preliminary results concerning (1.1). Section 3 is devoted to the proof of Theorem 1.1. 


\section{Preliminary results}

In this section we colect some useful results regarding problem (1.1). The first result in this sense is due to Dall'Acqua and Sweers [6, Theorem 12, Lemma C.2] and provides upper bounds for the Green function of the biharmonic operator subject to Dirichlet boundary conditions.

Proposition 2.1 (see [6]) Let $k$ be a n-dimensional multi-index. Then, there exists a positive constant $c$ depending on $\Omega$ and $k$ such that for any $x, y \in \Omega$ we have

(i) For $|k| \geq 2$ :

(i1) if $n>4-|k|$ then

$$
\left|D_{x}^{k} G(x, y)\right| \leq c|x-y|^{4-n-|k|} \min \left\{1, \frac{\delta(y)}{|x-y|}\right\}^{2}
$$

(i2) if $n=4-|k|$ then

$$
\left|D_{x}^{k} G(x, y)\right| \leq c \log \left(2+\frac{\delta(y)}{|x-y|}\right) \min \left\{1, \frac{\delta(y)}{|x-y|}\right\}^{2},
$$

(i3) if $n<4-|k|$ then

$$
\left|D_{x}^{k} G(x, y)\right| \leq c \delta(y)^{4-n-|k|} \min \left\{1, \frac{\delta(y)}{|x-y|}\right\}^{n+|k|-2} .
$$

(ii) For $|k|<2$ :

(ii1) if $n>4-|k|$ then

$$
\left|D_{x}^{k} G(x, y)\right| \leq c|x-y|^{4-n-|k|} \min \left\{1, \frac{\delta(x)}{|x-y|}\right\}^{2-|k|} \min \left\{1, \frac{\delta(y)}{|x-y|}\right\}^{2},
$$

(ii2) if $n=4-|k|$ then

$$
\left|D_{x}^{k} G(x, y)\right| \leq c \log \left(2+\frac{\delta(y)}{|x-y|}\right) \min \left\{1, \frac{\delta(x)}{|x-y|}\right\}^{2-|k|} \min \left\{1, \frac{\delta(y)}{|x-y|}\right\}^{2},
$$

(ii3) if $2(2-|k|) \leq n<4-|k|$ then

$$
\left|D_{x}^{k} G(x, y)\right| \leq c \delta(y)^{4-n-|k|} \min \left\{1, \frac{\delta(x)}{|x-y|}\right\}^{2-|k|} \min \left\{1, \frac{\delta(y)}{|x-y|}\right\}^{n+|k|-2},
$$

(ii4) if $n<2(2-|k|)$ then

$$
\left|D_{x}^{k} G(x, y)\right| \leq c \delta^{2-|k|-n / 2}(x) \delta^{2-n / 2}(y) \min \left\{1, \frac{\delta(x)}{|x-y|}\right\}^{n / 2} \min \left\{1, \frac{\delta(y)}{|x-y|}\right\}^{n / 2} .
$$

Let $\varphi_{1}$ be the first eigenfunction of $(-\Delta)$ in $H_{0}^{1}(\Omega)$. It is well known that $\varphi_{1}$ has constant sign in $\Omega$, so by a suitable normalization we may assume $\varphi_{1}>0$ in $\Omega$. Therefore, $\varphi_{1}$ satisfies

$$
\begin{cases}-\Delta \varphi_{1}=\lambda_{1} \varphi_{1}, \varphi_{1}>0 & \text { in } \Omega, \\ \varphi_{1}=0 & \text { on } \partial \Omega,\end{cases}
$$


where $\lambda_{1}>0$ is the first eigenvalue of $(-\Delta)$. By Hopf maximum principle [20] we have $\partial_{\nu} \varphi_{1}<0$ on $\partial \Omega$. Also, by the regularity of $\Omega$ we have $\varphi_{1} \in C^{4}(\bar{\Omega})$ and

$$
c \delta(x) \leq \varphi_{1}(x) \leq \frac{1}{c} \delta(x) \quad \text { in } \Omega,
$$

for some $0<c<1$.

Proposition 2.2 Let $u$ be a solution of problem (1.1). Then, there exist $c_{1}, c_{2}>0$ such that $u$ satisfies (1.3).

Proof. Let $a(x)=\varphi_{1}^{2}(x), x \in \bar{\Omega}$. It is easy to see that since $\varphi_{1} \in C^{4}(\bar{\Omega})$ then

$$
f:=\Delta^{2} a=2 \lambda_{1}^{2} \varphi_{1}^{2}+\sum_{i=1}^{n}\left[8 \frac{\partial \varphi_{1}}{\partial x_{i}} \frac{\partial^{3} \varphi_{1}}{\partial x_{i}^{3}}+6\left(\frac{\partial^{2} \varphi_{1}}{\partial x_{i}^{2}}\right)^{2}\right]
$$

is bounded in $\bar{\Omega}$, so, by the continuity of $u$ there exists $m>0$ small enough such that

$$
u(x)-m a(x)=\int_{\Omega} G(x, y)\left[u^{-\alpha}(y)-m f(y)\right] d y \geq 0 \quad \text { for all } x \in \Omega .
$$

Therefore,

$$
u(x) \geq \operatorname{ma}(x) \geq c_{0} \delta^{2}(x) \quad \text { in } \Omega,
$$

for some $c_{0}>0$. This proves the first part of the inequality in (1.3). For the second part, assume first $n>4$ and let $x \in \Omega$. Using Proposition 2.1(ii1), for all $y \in \Omega$ we have

$$
\begin{aligned}
G(x, y) & \leq c|x-y|^{2-n} \delta^{2}(x) \min \left\{1, \frac{\delta(y)}{|x-y|}\right\}^{2} \\
& \leq c|x-y|^{2-n} \delta^{2}(x) \min \left\{1, \frac{\delta(y)}{|x-y|}\right\}^{2 \alpha} \\
& =c|x-y|^{2-2 \alpha-n} \delta^{2}(x) \delta^{2 \alpha}(y) .
\end{aligned}
$$

Now, from (2.3) and (2.4) we have

$$
\begin{aligned}
u(x) & =\int_{\Omega} G(x, y) u^{-\alpha}(y) d y \\
& \leq c_{1} \int_{\Omega} G(x, y) \delta^{-2 \alpha}(y) d y \leq c_{2} \delta^{2}(x) \int_{\Omega}|x-y|^{2-2 \alpha-n} d y \\
& \leq c_{2} \delta^{2}(x) \int_{0 \leq|x-y| \leq \operatorname{diam}(\Omega)}|x-y|^{2-2 \alpha-n} d y \\
& =c_{2} \delta^{2}(x) \int_{0}^{\operatorname{diam}(\Omega)} t^{1-2 \alpha} d t \\
& \leq c_{3} \delta^{2}(x) .
\end{aligned}
$$

Assume now $n=4$. We use Proposition 2.1(ii2) to derive a similar inequality to (2.4). More precisely, for all $y \in \Omega$ we have

$$
\begin{aligned}
G(x, y) & \leq c \log \left(2+\frac{\delta(y)}{|x-y|}\right) \min \left\{1, \frac{\delta(x)}{|x-y|}\right\}^{2} \min \left\{1, \frac{\delta(y)}{|x-y|}\right\}^{2 \alpha} \\
& \leq c|x-y|^{-2-2 \alpha} \delta^{2}(x) \delta^{2 \alpha}(y) \log \left(2+\frac{\operatorname{diam}(\Omega)}{|x-y|}\right) .
\end{aligned}
$$


If $n=3$, let $\beta=\max \{0,2 \alpha-1 / 2\}<3 / 2$ and by Proposition 2.1(ii4) we have

$$
\begin{aligned}
G(x, y) & \leq c \delta^{1 / 2}(x) \delta^{1 / 2}(y) \min \left\{1, \frac{\delta(x)}{|x-y|}\right\}^{3 / 2} \min \left\{1, \frac{\delta(y)}{|x-y|}\right\}^{3 / 2} \\
& \leq c|x-y|^{-3 / 2-\beta} \delta^{2}(x) \delta^{\beta+1 / 2}(y) \\
& \leq C|x-y|^{-3 / 2-\beta} \delta^{2}(x) \delta^{2 \alpha}(y) .
\end{aligned}
$$

Finally, if $n=2$, let $\beta=\max \{0,2 \alpha-1\}<1$ and by Proposition 2.1(ii3) we have

$$
\begin{aligned}
G(x, y) & \leq c \delta(x) \delta(y) \min \left\{1, \frac{\delta(x)}{|x-y|}\right\} \min \left\{1, \frac{\delta(y)}{|x-y|}\right\} \\
& \leq c|x-y|^{-1} \delta^{2}(x) \delta(y) \min \left\{1, \frac{\delta(y)}{|x-y|}\right\}^{\beta} \\
& \leq c|x-y|^{-1-\beta} \delta^{2}(x) \delta^{1+\beta}(y) \\
& \leq C|x-y|^{-1-\beta} \delta^{2}(x) \delta^{2 \alpha}(y) .
\end{aligned}
$$

We now use the estimates (2.6)-(2.8) to derive a similar inequality to that in (2.5).

This completes the proof of Proposition 2.2.

Proposition 2.3 Let $0<\alpha<1$ and $u \in C(\bar{\Omega})$ be such that $u(x) \geq c \delta^{2}(x)$ in $\Omega$ for some $c>0$. Consider

$$
w(x)=\int_{\Omega} G(x, y) u^{-\alpha}(y) d y \quad \text { for all } x \in \bar{\Omega} .
$$

Then

(i) $w \in C^{2}(\bar{\Omega})$;

(ii) $w \in C^{3}(\bar{\Omega})$ for any $0<\alpha<1 / 2$.

Proof. With the same proof as in Proposition 2.2 it is easy to see that $v$ is well defined. For $0<\varepsilon<1$ small, define $\Omega_{\varepsilon}=\{x \in \bar{\Omega}: \delta(x)<\varepsilon\}$. Set

$$
u_{\varepsilon}(x)=\left\{\begin{array}{lr}
u(x) & \text { if } x \in \Omega \backslash \Omega_{\varepsilon}, \\
0 & \text { if } x \in \Omega_{\varepsilon} .
\end{array}\right.
$$

and

$$
w_{\varepsilon}(x)=\int_{\Omega} G(x, y) u_{\varepsilon}^{-\alpha}(y) d y \quad \text { for all } x \in \bar{\Omega} .
$$

Since $u_{\varepsilon}^{-\alpha}$ is bounded in $\bar{\Omega}$, by the estimates in Proposition 2.1 it follows that $w_{\varepsilon} \in C^{3}(\bar{\Omega})$ and

$$
D_{x}^{k} w_{\varepsilon}(x)=\int_{\Omega} D_{x}^{k} G(x, y) u_{\varepsilon}^{-\alpha}(y) d y \quad \text { for all } x \in \bar{\Omega},
$$

for any $n$-dimensional multi-index $k$ with $|k| \leq 3$. The proof of this fact is similar to that of Lemma 4.1 in [11]. We employ in the following the same approach as in [11] to show that $w \in C^{2}(\bar{\Omega})\left(\right.$ resp. $w \in C^{3}(\bar{\Omega})$ if $0<\alpha<1 / 2$ ).

Assume first that $n>4$ and let $k$ be a $n$-dimensional multi-index with $|k| \leq 2$. Fix $\beta>0$ such that $2 \alpha<\beta<2$. 
By Proposition 2.1(i1) (if $|k|=2$ ) and (ii1) (if $|k| \leq 1$ ) we have

$$
\begin{aligned}
& \left|D_{x}^{k} w_{\varepsilon}(x)-\int_{\Omega} D_{x}^{k} G(x, y) u^{-\alpha}(y) d y\right| \leq \int_{\Omega_{\varepsilon}}\left|D_{x}^{k} G(x, y)\right| u^{-\alpha}(y) d y \\
& \leq c_{1} \int_{\Omega_{\varepsilon}}|x-y|^{4-|k|-n} \delta^{-2 \alpha}(y) \min \left\{1, \frac{\delta(y)}{|x-y|}\right\}^{2} d y \\
& \leq c_{1} \int_{\Omega_{\varepsilon}}|x-y|^{4-|k|-n} \delta^{-2 \alpha}(y) \min \left\{1, \frac{\delta(y)}{|x-y|}\right\}^{\beta} d y \\
& \leq c_{1} \int_{\Omega_{\varepsilon}}|x-y|^{4-|k|-\beta-n} \delta^{\beta-2 \alpha}(y) d y \\
& \leq c_{1} \varepsilon^{\beta-2 \alpha} \int_{\Omega}|x-y|^{4-|k|-\beta-n} d y \\
& \leq c_{1} \varepsilon^{\beta-2 \alpha} \int_{0 \leq|x-y| \leq \operatorname{diam}(\Omega)}|x-y|^{4-|k|-\beta-n} d y \\
& \leq c_{1} \varepsilon^{\beta-2 \alpha} \int_{0}^{\operatorname{diam}(\Omega)} t^{3-|k|-\beta} d t \\
& \leq c_{2} \varepsilon^{\beta-2 \alpha} \int_{0}^{\operatorname{diam}(\Omega)} t^{1-\beta} d t \leq c_{3} \varepsilon^{\beta-2 \alpha} \rightarrow 0 \quad \text { as } \varepsilon \rightarrow 0 .
\end{aligned}
$$

The case $2 \leq n \leq 4$ can be analyzed in the same way. For instance, if $n=3$ and $|k|=1$, we use Proposition 2.1(ii2) to derive

$$
\begin{aligned}
\left|D_{x}^{k} w_{\varepsilon}(x)-\int_{\Omega} D_{x}^{k} G(x, y) u^{-\alpha}(y) d y\right| & \leq c_{1} \int_{\Omega_{\varepsilon}} \log \left(2+\frac{\delta(y)}{|x-y|}\right) \delta^{-2 \alpha}(y) \min \left\{1, \frac{\delta(y)}{|x-y|}\right\}^{2} d y \\
& \leq c_{1} \int_{\Omega_{\varepsilon}}|x-y|^{-\beta} \log \left(2+\frac{\delta(y)}{|x-y|}\right) \delta^{\beta-2 \alpha}(y) d y \\
& \leq c_{1} \varepsilon^{\beta-2 \alpha} \int_{\Omega_{\varepsilon}}|x-y|^{-\beta} \log \left(2+\frac{\operatorname{diam}(\Omega)}{|x-y|}\right) d y \\
& \leq c_{2} \varepsilon^{\beta-2 \alpha} \int_{0}^{\operatorname{diam}(\Omega)} t^{2-\beta} \log \left(2+\frac{\operatorname{diam}(\Omega)}{t}\right) d t \\
& \leq c_{3} \varepsilon^{\beta-2 \alpha} \rightarrow 0 \text { as } \varepsilon \rightarrow 0 .
\end{aligned}
$$

We have obtained that

$$
D_{x}^{k} w_{\varepsilon} \rightarrow \int_{\Omega} D_{x}^{k} G(\cdot, y) u^{-\alpha}(y) d y \quad \text { uniformly as } \varepsilon \rightarrow 0,
$$

for any $n$-dimensional multi-index $k$ with $0 \leq|k| \leq 2$. It follows that $w \in C^{2}(\bar{\Omega})$ and

$$
D_{x}^{k} w(x)=\int_{\Omega} D_{x}^{k} G(x, y) u^{-\alpha}(y) d y \quad \text { for all } x \in \bar{\Omega},
$$

for any multi-index $k$ with $0 \leq|k| \leq 2$. 
(ii) Let $k$ be a multi-index with $|k|=3$ and $2 \alpha<\beta<1$. From Proposition 2.1(i1) we have

$$
\begin{aligned}
\left|D_{x}^{k} w_{\varepsilon}(x)-\int_{\Omega} D_{x}^{k} G(x, y) u^{-\alpha}(y) d y\right| & \leq \int_{\Omega_{\varepsilon}}\left|D_{x}^{k} G(x, y)\right| u^{-\alpha}(y) d y \\
& \leq c_{1} \int_{\Omega_{\varepsilon}}|x-y|^{1-n} \delta^{-2 \alpha}(y) \min \left\{1, \frac{\delta(y)}{|x-y|}\right\}^{\beta} d y \\
& \leq c_{1} \varepsilon^{\beta-2 \alpha} \int_{\Omega}|x-y|^{1-n-\beta} d y \\
& \leq c_{1} \varepsilon^{\beta-2 \alpha} \int_{0}^{\operatorname{diam}(\Omega)} t^{-\beta} d t \leq c_{2} \varepsilon^{\beta-2 \alpha} \rightarrow 0 \quad \text { as } \varepsilon \rightarrow 0,
\end{aligned}
$$

since $\beta<1$. With the same arguments as above we find $w \in C^{3}(\bar{\Omega})$. This completes the proof.

\section{Proof of Theorem 1.1}

Let $a(x)=\varphi_{1}^{2}(x), x \in \bar{\Omega}$. Motivated by Proposition 2.2 we will be looking for solutions $u$ of (1.1) in the form

$$
u(x)=a(x) v(x)
$$

where $v \in C(\bar{\Omega}), v>0$ in $\bar{\Omega}$. This leads us to the following integral equation for $v$ :

$$
v(x)=\frac{1}{a(x)} \int_{\Omega} \frac{G(x, y)}{a^{\alpha}(y)} v^{-\alpha}(y) d y \quad \text { for all } x \in \bar{\Omega} .
$$

We can now regard (3.1) as the fixed point problem

$$
\mathscr{F}(v)=v,
$$

where

$$
\mathscr{F}(v)=\frac{1}{a(x)} \int_{\Omega} \frac{G(x, y)}{a^{\alpha}(y)} v^{-\alpha}(y) d y .
$$

Remark that $\mathscr{F}$ is an integral operator of the form

$$
\mathscr{F}(v)=\int_{\Omega} K(x, y) v^{-\alpha}(y) d y,
$$

where the kernel $K$ is given by

$$
K: \bar{\Omega} \times \Omega \rightarrow[0, \infty], \quad K(x, y)= \begin{cases}\frac{G(x, y)}{a(x) a^{\alpha}(y)} & \text { if } x, y \in \Omega, \\ \frac{\partial_{\nu}^{2} G(x, y)}{\partial_{\nu}^{2} a(x) a^{\alpha}(y)} & \text { if } x \in \partial \Omega, y \in \Omega .\end{cases}
$$

Note that $K$ is well defined since $\partial_{\nu}^{2} a(x)=2\left(\partial_{\nu} \varphi_{1}(x)\right)^{2}>0$ on $\partial \Omega$.

We first need the following result.

Lemma 3.1 (i) For any $y \in \Omega$, the function $K(\cdot, y): \bar{\Omega} \rightarrow[0, \infty]$ is continuous;

(ii) The mapping

$$
\bar{\Omega} \ni x \mapsto \int_{\Omega} K(x, y) d y
$$

is continuous and there exists $M>1$ such that

$$
\frac{1}{M} \leq \int_{\Omega} K(x, y) d y \leq M \quad \text { for all } x \in \bar{\Omega} .
$$


Proof. Since the Green function is continuous on $\Omega \times \Omega$, it remains to prove the continuity of $K(\cdot, y)$ on $\partial \Omega$. Let $\varepsilon>0$. Since $G(\cdot, y) \in C^{4}(\bar{\Omega} \backslash\{y\})$ and $a \in C^{4}(\bar{\Omega})$, for any $z \in \partial \Omega$ we have

$$
\begin{aligned}
& G(z+t \nu, y)=t^{2}\left(\frac{1}{2} \partial_{\nu}^{2} G(z, y)+G_{1}(z, t)\right) \quad \text { as } t \nearrow 0, \\
& a(z+t \nu, y)=t^{2}\left(\frac{1}{2} \partial_{\nu}^{2} a(z, y)+a_{1}(z, t)\right) \quad \text { as } t \nearrow 0,
\end{aligned}
$$

where

$$
\lim _{t \nearrow 0} G_{1}(z, t)=\lim _{t \nearrow 0} a_{1}(z, t)=0 \quad \text { uniformly for } z \in \partial \Omega .
$$

Hence, as $t \nearrow 0$ we have

$$
\begin{aligned}
|K(z+t \nu, y)-K(z, y)| & =\left|\frac{\frac{1}{2} \partial_{\nu}^{2} G(z, y)+G_{1}(z, t)}{\frac{1}{2} \partial_{\nu}^{2} a(z, y)+a_{1}(z, t)}-\frac{\partial_{\nu}^{2} G(z, y)}{\partial_{\nu}^{2} a(z, y)}\right| \\
& \leq \frac{\left|G_{1}(z, y)\right| \partial_{\nu}^{2} a(z, y)+\left|a_{1}(z, t)\right|\left|\partial_{\nu}^{2} G(z, y)\right|}{\partial_{\nu}^{2} a(z, y)\left|\frac{1}{2} \partial_{\nu}^{2} a(z, y)+a_{1}(z, t)\right|} .
\end{aligned}
$$

Thus, there exists $\eta_{1}>0$ such that

$$
|K(z+t \nu, y)-K(z, y)|<\frac{\varepsilon}{2} \quad \text { for all } z \in \partial \Omega \text { and }-\eta_{1}<t<0 .
$$

Also, by the smoothness of the boundary $\partial \Omega$ there exists $\eta_{2}>0$ such that

$$
|K(z, y)-K(\bar{z}, y)|<\frac{\varepsilon}{2} \quad \text { for all } z, \bar{z} \in \partial \Omega,|z-\bar{z}|<\eta_{2} .
$$

Define $\eta=\min \left\{\eta_{1}, \eta_{2}\right\} / 2$ and fix $z \in \partial \Omega$. Let now $x \in \bar{\Omega}$ be such that $|x-z|<\eta$. Also, let $\bar{x} \in \partial \Omega$ be such that $|x-\bar{x}|=\delta(x)=\operatorname{dist}(x, \partial \Omega)$. Then $|x-\bar{x}| \leq|x-z|<\eta$ and $|\bar{x}-z| \leq|x-\bar{x}|+|z-x|<2 \eta<\eta_{2}$ so by (3.4) we have

$$
|K(\bar{x}, y)-K(z, y)|<\frac{\varepsilon}{2} .
$$

Now, from (3.3) and (3.5) we obtain

$$
|K(x, y)-K(z, y)| \leq|K(x, y)-K(\bar{x}, y)|+|K(\bar{x}, y)-K(z, y)|<\varepsilon
$$

so $K(\cdot, y)$ is continuous at $z \in \partial \Omega$. This completes the proof of (i).

(ii) Assume first $n>4$. Using (2.2) and Proposition 2.1(ii1) we have

$$
\begin{aligned}
K(x, y) & \leq c_{1} \delta^{-2}(x) \delta^{-2 \alpha}(y) G(x, y) \\
& \leq c_{2}|x-y|^{2-n} \delta^{-2 \alpha}(y) \min \left\{1, \frac{\delta(y)}{|x-y|}\right\}^{2} \\
& \leq c_{2}|x-y|^{2-n} \delta^{-2 \alpha}(y) \min \left\{1, \frac{\delta(y)}{|x-y|}\right\}^{2 \alpha} \\
& \leq c_{2}|x-y|^{2-2 \alpha-n} \quad \text { for all } x, y \in \Omega .
\end{aligned}
$$

Since $0<\alpha<1$, the mapping $x \mapsto|x-y|^{2-2 \alpha-n}$ is integrable on $\Omega$, so by means of Lebesgue's dominated convergence Theorem we deduce that $\bar{\Omega} \ni x \mapsto \int_{\Omega} K(x, y) d y$ is continuous. This fact combined with $K>0$ in $\Omega$ proves the existence of a number $M>1$ that satisfies (3.2). 
For $2 \leq n \leq 4$ we proceed similarly with different estimates (as in the proof of Proposition $2.2)$ to derive the same conclusion.

Let $M>1$ satisfy (3.2) and fix $0<\varepsilon<1$ such that

$$
\varepsilon^{1-\alpha^{2}} \leq M^{-1-\alpha}
$$

Define

$$
g_{\varepsilon}: \mathbb{R} \rightarrow \mathbb{R}, \quad g_{\varepsilon}(t)= \begin{cases}\varepsilon^{-\alpha} & \text { if } t<\varepsilon, \\ t^{-\alpha} & \text { if } t \geq \varepsilon,\end{cases}
$$

and for any $v \in C(\bar{\Omega}), v>0$ in $\bar{\Omega}$ consider the operator

$$
T_{\varepsilon}(v)(x)=\int_{\Omega} K(x, y) g_{\varepsilon}(v(y)) d y \quad \text { for all } x \in \bar{\Omega} .
$$

If $v \in C(\bar{\Omega})$ satisfies $v>0$ in $\bar{\Omega}$, then $g_{\varepsilon}(v) \leq \varepsilon^{-\alpha}$ in $\bar{\Omega}$ so by (3.2) we find $T_{\varepsilon}(v) \leq M \varepsilon^{-\alpha}$ in $\bar{\Omega}$. Let now

$$
v_{1} \equiv M^{-1-\alpha} \varepsilon^{\alpha^{2}}, \quad v_{2} \equiv M \varepsilon^{-\alpha} .
$$

and

$$
\left[v_{1}, v_{2}\right]=\left\{v \in C(\bar{\Omega}): v_{1} \leq v \leq v_{2}\right\} .
$$

By Lemma (3.2) it is easy to see that $T_{\varepsilon}\left(\left[v_{1}, v_{2}\right]\right) \subseteq\left[v_{1}, v_{2}\right]$. Further, by Lemma 3.1 and ArzelaAscoli theorem, it follows that

$$
T_{\varepsilon}:\left[v_{1}, v_{2}\right] \rightarrow\left[v_{1}, v_{2}\right] \quad \text { is compact. }
$$

Hence, by Schauder fixed point theorem, there exists $v \in C(\bar{\Omega}), v_{1} \leq v \leq v_{2}$ in $\bar{\Omega}$ such that $T_{\varepsilon}(v)=v$. By (3.6) it follows that $v \geq v_{1} \geq \varepsilon$ in $\bar{\Omega}$, so $g_{\varepsilon}(v)=v^{-\alpha}$. Therefore, $v$ satisfies (3.1), that is, $u=a v$ is a solution of (1.1). Now, the the boundary estimate (1.3) and the regularity of solution $u$ follows from Proposition 2.2 and Proposition 2.3 respectively. In the following we derive the uniqueness of the solution to (1.1).

Let $u_{1}, u_{2}$ be two solutions of (1.1). Using Proposition 2.2 there exists $0<c<1$ such that

$$
c \delta^{2}(x) \leq u_{i}(x) \leq \frac{1}{c} \delta^{2}(x) \quad \text { in } \Omega, i=1,2 .
$$

This means that we can find a constant $C>1$ such that $C u_{1} \geq u_{2}$ and $C u_{2} \geq u_{1}$ in $\Omega$.

We claim that $u_{1} \geq u_{2}$ in $\Omega$. Supposing the contrary, let

$$
M=\inf \left\{A>1: A u_{1} \geq u_{2} \text { in } \Omega\right\} .
$$

By our assumption, we have $M>1$. From $M u_{1} \geq u_{2}$ in $\Omega$, it follows that

$$
M^{\alpha} u_{2}(x)-u_{1}(x)=\int_{\Omega} G(x, y)\left[M^{\alpha} u_{2}^{-\alpha}(y)-u_{1}^{-\alpha}(y)\right] d y \geq 0 \quad \text { for all } x \in \Omega,
$$

and then

$$
M^{\alpha^{2}} u_{1}(x)-u_{2}(x)=\int_{\Omega} G(x, y)\left[M^{\alpha^{2}} u_{1}^{-\alpha}(y)-u_{2}^{-\alpha}(y)\right] d y \geq 0 \quad \text { for all } x \in \Omega .
$$

We have thus obtained $M^{\alpha^{2}} u_{1} \geq u_{2}$ in $\Omega$. Since $M>1$ and $\alpha^{2}<1$, this last inequality contradicts the minimality of $M$. Hence, $u_{1} \geq u_{2}$ in $\Omega$. Similarly we deduce $u_{1} \leq u_{2}$ in $\Omega$, so $u_{1} \equiv u_{2}$ and the uniqueness is proved. This finishes the proof of Theorem 1.1. 


\section{References}

[1] G. Arioli, F. Gazzola, H.-Ch. Grunau and E. Mitidieri, A semilinear fourth order elliptic problem with exponential nonlinearity, SIAM J. Math. Anal. 36 (2005), 1226-1258.

[2] T. Boggio, Sull'equilibrio delle piastre elastiche incastrate, Rend. Acc. Lincei 10 (1901), 197-205.

[3] T. Boggio, Sulle funzioni di Green d'ordine m, Rend. Circ. Mat. Palermo 20 (1905), 97-135.

[4] C.V. Coffman and R. J. Duffin, On the structure of biharmonic functions satisfying the clamped condition on a right angle, Adv. Appl. Math. 1 (1950), 373-389.

[5] C. V. Coffman and C. L. Grover, Obtuse cones in Hilbert spaces and applictions to partial differential equations, J. Funct. Anal. 35 (1980), 369-396.

[6] A. Dall'Acqua and G. Sweers, Estimates for Green function and Poisson kernels of higher order Dirichlet boundary value problems, J. Differ. Equations 205 (2004), 466-487.

[7] A. Ferrero and H.-Ch. Grunau, The Dirichlet problem for supercritical biharmonic equations with power-type nonlinearity, J. Differ. Equations 234 (2007), 582-606.

[8] P.R. Garabedian, A partial differential equation arising in conformal mapping, Pacific J. Math. 1 (1951), 485-524.

[9] F. Gazzola, H.-Ch. Grunau and M. Squassina, Existence and nonexistence results for critical growth biharmonic elliptic equations, Calc. Var. PDE 18 (2003), 117-143.

[10] F. Gazzola and H.-Ch. Grunau, Radial entire solutions for supercritical biharmonic equations, Math. Ann. 334 (2006), 905-936.

[11] D. Gilbarg and N.S. Trudinger, Elliptic Partial Differential Equations of Second Order, Springer, 3rd Ed. 2001.

[12] H.-Ch. Grunau and G. Sweers, Positivity for perturbations of polyharmonic operators with Dirichlet boundary conditions in two dimensions, Math. Nachr. 179 (1996), 89-102.

[13] H.-Ch. Grunau and G. Sweers, Positivity for equations involving polyharmonic operators with Dirichlet boundary conditions, Math. Ann. 307 (1997), 589-626.

[14] H.-Ch. Grunau and G. Sweers, Sign change for the Green function and for the first eigenfunction of equations of clamped plate type, Archive Rational Mech. Anal. 150 (1999), 179-190.

[15] H.-Ch. Grunau and G. Sweers, Regions of positivity for polyharmonic Green functions in arbitrary domains, Proc. Amer. Math. Soc. 135 (2007), 3537-3546.

[16] H.-Ch. Grunau and F. Robert, Positivity and almost positivity of biharmonic Green's functions under Dirichlet boundary conditions, Arch. Rational Mech. Anal., in press.

[17] J. Hadamard, Mémoire sur le problème d'analyse relatif à l'équilibre des plaques élastiques encastrées, in: OEuvres de Jacques Hadamard, Tome II, 515-641, CNRS: Paris (1968).

[18] B. Kawohl and G.Sweers, On anti-eigenvalues for elliptic systems and a question of McKenna and Walter, Indiana Univ. Math. J. 51 (2002), 1023-1040. 
[19] J.P. Krasovskiul, Isolation of singularities of the Green function (Russian), Izv. Akad. Nauk SSSR Ser. Mat. 31 (1967), 977-1010, English translation in: Math. USSR, Izv. 1 (1967), 935-966.

[20] M.H. Protter, H.F. Weinberger, Maximum Principles in Differential Equations, Prentice Hall, Englewood Cliffs N.J., 1967.

[21] H.S. Shapiro and M. Tegmark, An elementary proof that the biharmonic Green function of an eccentric ellipse changes sign, SIAM Rev. 36 (1994), 99-101. 